\title{
Remote controller using steady state visual evoked potential ${ }^{*}$
}

\author{
Song Cheng, Mei Wang*, Liang Wang, \\ Hong-Guang Pan and Song-Zhi Zhang \\ College of Electrical and control engineering, \\ Xi'an University of Science \&Technology, 7l0054, Xi'an, China \\ *Corresponding author, E-mail: wangm@qq.com
}

\begin{abstract}
This paper develops a new air-conditioning remote ccontrol system which based on brain waves and the Android system. First of all, the desired brain waves in EEG signals in the form of steady-state visual evoked is collected. By extracting and analysis the feature hidden in EEG signal, the system can identify the operation intention of volunteer to control air-conditioning machine, such as open or close and addition or subtraction about temperature. In addition, the control coding and wireless connections are implemented based on Bluetooth technology. Finally, we realize the Android mobile phones as the core of whole remote control. The experimental results show that brain wave control system of air conditioner remote is capable of stable and rapid detection of brain signals to control commands. Compared to commonly used buttons remote control, our designed system can realize the goal of non-contact control for air-conditioning.
\end{abstract}

Keywords: EEG; Brain wave; Android system; BCI; air-conditioning.

\section{Introduction}

In order to achieve the goal of facilitating patients with severe disability a new life style, making them live independently by non-contact and no muscle action, developing a novel way to innovate the traditional human-computer interaction manner is becoming more and more fascinating [1-5].

In this paper, we made a research on the construction and extraction of brain waves control command, designed and realized the simple control of air conditioning based on BCI and infrared technology.

\section{System Design}

The acquisition of EEG signal is mainly through NeuroSky chip, which can pretreat and sample potential of the occipital lobe, it collect the EEG potential of

\footnotetext{
${ }^{*}$ This work is supported by Natural Science Foundation of China (61603295),Key Scientific and Technological Project of Shaanxi Province (2016GY-040), and Science Foundation of Xi'an University of Science and Technology (104-6319900001)
} 
the occipital area through an electrode. This process is common and noninvasive for the EEG acquisition, because it only requires the volunteers to wear a electrode cap on the scalp. When the sampling process is finished, the signal will be sent by NeuroSky's TXD pin to BlueTooth's RX pin and Android. Specifically, Android is responsible for processing of the brain wave data analysis and feature extraction. According to the frequency of the signal in its current state, system can determine what the user wants to do , and send control signal to ARM.

\subsection{EEG denoising based on wavelet decomposition and reconstruction}

In order to get the user's intention, we remove noise from the original brain waves as shown in Fig. 2 by using wavelet decomposition and reconstruction. In this process, the high frequency interference signal of $50 \mathrm{~Hz}$ is mainly investigated to filter the noise data from original EEG information.

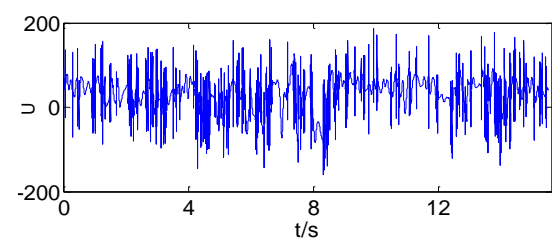

(a)

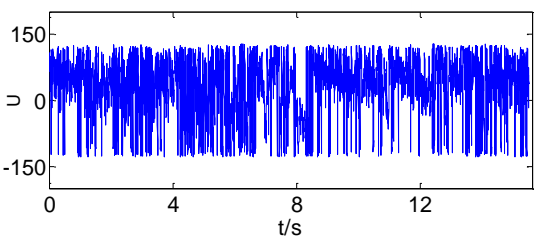

(b)

Fig. 2 (a) original brain wave signal and (b) brain wave signal after denoising.

\subsection{Extraction of brainwave control}

The common methods of dealing message are independent vector based method, Fourier transforms method, and wavelet transforms method and so on [6-15]. Given the instantaneity of developed system, we use FFT to transform the EEG signal from time domain to frequency domain, and extract the corresponding features in frequency domain. Afterward, the identified features will be leveraged to classify the brain wave. The final obtained frequency spectrum of EEG is shown in Fig. 3(a) and Fig. 3(b).Next; we elaborate the modified FFT from DFT eq. (1). Set $\mathrm{N}$ ( $\mathrm{N}=2 \mathrm{M}, \mathrm{M}$ equal positive integer) points message sequence $X(n)$ transformed by DFT, and get $X(k)$, so get:

$$
\begin{aligned}
& X(k)=\sum_{k=0}^{N-1} x(n) W_{N}^{n k} \\
& x(n)=\frac{1}{N} \sum_{k=0}^{N-1} X(k) W_{N}^{-n k}
\end{aligned}
$$


$n=0,1 \ldots \mathrm{N}-1, \mathrm{k}=0,1 \ldots \mathrm{N}-1$, and classify $\mathrm{x}(\mathrm{n})(\mathrm{n}=0,1 \ldots \mathrm{N}-1)$ to two grounds by parity:

$$
\left\{\begin{array}{l}
x(2 r)=x_{1}(r) \\
x(2 r+1)=x_{2}(r)
\end{array} \quad r=0,1, \cdots \frac{N}{2}-1\right.
$$

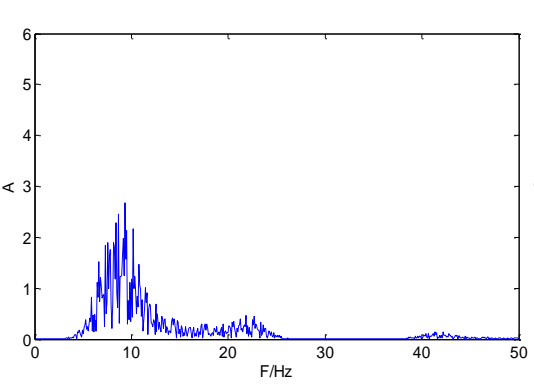

(a)

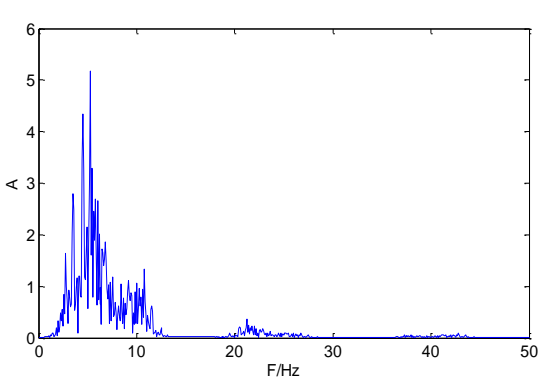

(b)

Fig. 3. $6 \mathrm{~Hz}$ frequency spectrum (a) and $9 \mathrm{~Hz}$ frequency spectrum (b)

If we bring the eq. (3) into eq. (1), and simplify it by periodicity and symmetry, then the below equation can be drived:

$$
\begin{array}{r}
X(k)=X_{1}(k)+W_{N}^{K} X_{2}(k) \\
X\left(k+\frac{N}{2}\right)=X_{1}(k)+W_{N}^{K} X_{2}(k)
\end{array}
$$

So $\mathrm{N}$ point sequence into two $\mathrm{N} / 2$ point sequence $\mathrm{x}_{1}(\mathrm{r})$ and $\mathrm{x}_{2}(\mathrm{r})$ and the combination of the discrete Fourier transform. Among them,

$$
X_{1}(k)=\sum_{r=0}^{N / 2-1} x_{1}(r) W_{N / 2}^{r k}, X_{2}(k)=\sum_{r=0}^{N / 2-1} x_{2}(r) W_{N / 2}^{r k}, W_{N}^{n k}=e^{-j \frac{2 \pi}{N} n k}
$$

This decomposition will be repeated, until the basic transform reaches two point.

Applying FFT transform one second message in the time domain, we can get 512 amplitudes by $512 \mathrm{~Hz}$ sample frequency. The resultant of experiment is shown in Fig. 3(a) and Fig. 3(b). Research of Tsinghua University shows that it will recommend to adopt $7-12 \mathrm{~Hz}$ frequency in experiment. Our designed system use $6 \mathrm{~Hz}, 7 \mathrm{~Hz}, 9 \mathrm{~Hz}$, and $11 \mathrm{~Hz}$, as characteristics to classify different order. 


\section{Experiment and Result Analysis}

\subsection{Experimental Environment}

Padfone of ASUS, STM32F103VET6 is a kind of ARM controller and air conditioner of Medea. As for the tested data set, 5 student participants with normal visual acuity are selected, who are male with the odd number and female with the even numbers between the ages of 20-26 years old. The evaluation metrics of the experimental results are time and error rate. Each person testes the function of "open", "off", "temperature", "mode" three times. The interval of each test is 1 hour.

\subsection{Experimental Results and Analysis}

Five volunteers who have never been trained are chosen to implement the experiments... The results of the experiment, under the condition of of $6 \mathrm{~Hz}, 7 \mathrm{~Hz}$, $9 \mathrm{~Hz}, 11 \mathrm{~Hz}$, are as follows: Table 1, Table 2, Table 3 and Table 4. Among them, $\mathrm{T} 1, \mathrm{~T} 2$, T3 represent three different test, while is the average time of these three times, ER indicates the error rate.

Table $16 \mathrm{HZ}$ and $7 \mathrm{HZ}$ functional test

\begin{tabular}{ccccccccccc}
\hline & \multicolumn{1}{c}{6} & \multicolumn{1}{c}{7} & \multicolumn{1}{c}{7} \\
\cline { 2 - 10 } & $\mathrm{T}_{1}$ & $\mathrm{~T}_{2}$ & $\mathrm{~T}_{3}$ & $\mathrm{~T}_{\mathrm{A}}$ & $\mathrm{E}_{\mathrm{R}}(\%)$ & $\mathrm{T}_{1}$ & $\mathrm{~T}_{2}$ & $\mathrm{~T}_{3}$ & $\mathrm{~T}_{\mathrm{A}}$ & $\mathrm{E}_{\mathrm{R}}(\%)$ \\
\hline 1 & 56 & 45 & 68 & 56.3 & 25.5 & 80 & 74 & 65 & 73.0 & 23.3 \\
2 & 48 & 55 & 60 & 54.3 & 27.4 & 76 & 65 & 81 & 74.0 & 20.4 \\
3 & 33 & 58 & 79 & 56.3 & 22.5 & 73 & 74 & 90 & 79.0 & 19.2 \\
4 & 57 & 47 & 69 & 57.7 & 21.7 & 65 & 66 & 87 & 72.7 & 20.1 \\
5 & 80 & 67 & 57 & 68.0 & 23.7 & 78 & 67 & 82 & 75.7 & 15.4 \\
\hline
\end{tabular}

Table $29 \mathrm{HZ}$ and $11 \mathrm{HZ}$ functional test

\begin{tabular}{|c|c|c|c|c|c|c|c|c|c|c|}
\hline & \multicolumn{5}{|c|}{9} & \multicolumn{5}{|c|}{11} \\
\hline & $\mathrm{T}_{1}$ & $\mathrm{~T}_{2}$ & $\mathrm{~T}_{3}$ & $\mathrm{~T}_{\mathrm{A}}$ & $\mathrm{E}_{\mathrm{R}}(\%)$ & $\mathrm{T}_{1}$ & $\mathrm{~T}_{2}$ & $\mathrm{~T}_{3}$ & $\mathrm{~T}_{\mathrm{A}}$ & $\mathrm{E}_{\mathrm{R}}(\%)$ \\
\hline 1 & 55 & 45 & 56 & 52.0 & 16.4 & 46 & 34 & 32 & 37.3 & 10.4 \\
\hline 2 & 54 & 43 & 51 & 49.3 & 18.1 & 45 & 42 & 50 & 45.7 & 15.6 \\
\hline 3 & 33 & 54 & 56 & 47.6 & 22.5 & 33 & 43 & 21 & 32.3 & 15.5 \\
\hline 4 & 45 & 39 & 47 & 43.7 & 21.7 & 36 & 43 & 42 & 40.3 & 16.4 \\
\hline 5 & 50 & 56 & 57 & 54.3 & 19.7 & 45 & 31 & 20 & 32.0 & 9.5 \\
\hline
\end{tabular}

The tested persons are trained by daily visual evoked once a day in two weeks. After that, the results are as recorded as follows: 
Table $36 \mathrm{HZ}$ and $7 \mathrm{HZ}$ functional test after training

\begin{tabular}{ccccccccccc}
\hline & \multicolumn{1}{c}{6} & & & & 7 & \\
\cline { 2 - 10 } & $\mathrm{T}_{1}$ & $\mathrm{~T}_{2}$ & $\mathrm{~T}_{3}$ & $\mathrm{~T}_{\mathrm{A}}$ & $\mathrm{E}_{\mathrm{R}}(\%)$ & $\mathrm{T}_{1}$ & $\mathrm{~T}_{2}$ & $\mathrm{~T}_{3}$ & $\mathrm{~T}_{\mathrm{A}}$ & $\mathrm{E}_{\mathrm{R}}(\%)$ \\
\hline 1 & 45 & 40 & 56 & 47.0 & 15.0 & 50 & 64 & 65 & 59.7 & 14.3 \\
2 & 43 & 38 & 37 & 39.3 & 15.5 & 66 & 60 & 66 & 64.0 & 13.4 \\
3 & 34 & 45 & 44 & 41.3 & 15.0 & 53 & 64 & 60 & 59.0 & 15.2 \\
4 & 51 & 45 & 57 & 51.0 & 14.5 & 65 & 56 & 66 & 62.3 & 13.1 \\
5 & 50 & 56 & 56 & 54.0 & 12.3 & 58 & 57 & 52 & 55.7 & 14.4 \\
\hline
\end{tabular}

Table 4. $9 \mathrm{HZ}$ and $11 \mathrm{HZ}$ functional test after training

\begin{tabular}{ccccccccccc}
\hline & \multicolumn{9}{c}{9} & \multicolumn{7}{c}{11} & \\
\cline { 2 - 10 } & $\mathrm{T}_{1}$ & $\mathrm{~T}_{2}$ & $\mathrm{~T}_{3}$ & $\mathrm{~T}_{\mathrm{A}}$ & $\mathrm{E}_{\mathrm{R}}(\%)$ & $\mathrm{T}_{1}$ & $\mathrm{~T}_{2}$ & $\mathrm{~T}_{3}$ & $\mathrm{~T}_{\mathrm{A}}$ & $\mathrm{E}_{\mathrm{R}}(\%)$ \\
\hline 1 & 35 & 25 & 36 & 32.0 & 11.4 & 26 & 34 & 22 & 27.3 & 8.4 \\
2 & 34 & 33 & 31 & 39.3 & 11.1 & 25 & 32 & 20 & 25.7 & 5.6 \\
3 & 34 & 30 & 44 & 36.0 & 12.0 & 23 & 23 & 11 & 12.0 & 5.5 \\
4 & 31 & 35 & 37 & 34.3 & 13.5 & 16 & 23 & 22 & 20.3 & 6.4 \\
5 & 40 & 25 & 36 & 33.0 & 12.3 & 25 & 21 & 20 & 22.0 & 7.5 \\
\hline
\end{tabular}

\section{Conclusion}

This paper designed a set of household used air-conditioning system based on brainwave control combined with BCI and infrared technology. Firstly, the weak signal in the occipital region of the human brain is collected. And then, by using FFT technology, the brain wave signal frequency spectrum characteristics can be obtained after preprocessing of noise removal. The infrared decoding on air conditioning is conducted in order to get the command signal of various functions. Afterward, based on the brain machine interface technology, many control functions can be achieved. Moreover, we analyze the related influence factors, such as man, woman, training or not and so on. Experiment shows that our designed system has the virtue of fast stability, simple construction, low cost, easy operation, good interface of human-computer interaction, high practical value and so on.

\section{References}

1. J. R. Wolpaw , N. Birbaumer, D. J. McFarland, et al. Brain-computer interfaces for communication and control. J.Clinical Neurophysiology, 113(6):767-791(2002).

2. Y. Q Li, J. Y Long, T.Y Yu, et al. An EEG-based BCI System for 2-D Cursor Control by Combining Mu/Beta Rhythm and P300 Potential. J. Biomedical Engineering, 57(10):2495-505(2010).

3. A. Lopez and P. C. Richardson. Capcitive electrocardiographic and bioelectric electrodes. IEEE Transactions on Biomedical Engineering, 16:299-300(2006). 
4. U.R. Acharya, S.V. Sree, G. Swapna, R.J. Martis, J.S. Suri, Automated EEG analysis of epilepsy: a review. Knowl-Based Syst. 45,pp. 147165(2013).

5. J. Gotman, P. Gloor, Automatic recognition and quantification of interictal epileptic activity in the human scalp EEG. Electroencephalogr. Clin. Neurophysiol. 41, pp. 513-529(2006).

6. R. Dhiman, J.S. Saini, Priyanka, Genetic algorithms tuned expert model for detection of epilepticseizures from EEG signatures. Appl. Soft. Comput. 19, pp. 8-17(2014).

7. Y. Kumar, M.L. Dewal, R.S. Anand, Epileptic seizure detection using DWT based fuzzy approximate entropy and support vector machine. Neuro computing .133, pp. 271-279 (2014).

8. R. Sharma, R.B. Pachori, Classification of epileptic seizures in EEG signals based on phase space representation of intrinsic mode functions, Expert. Syst. Appl. 42, pp. 1106-1117(2015).

9. Y. Zhang, G. X. Zhou, J. Jin, M. J Wang, X. Y Wang. L1-regularized multiway canonical correlation analysis for SSVEP-based BCI. IEEE Trans. Neural Syst. Rehabil. Eng. 21, pp. 887-896(2013).

10. G. Y. Bin, Y. J Zhang, X. R. Gao. Phase synchronization method for steady state visual evoked potential measurement. Journal of Tsinghua University, 48, pp. (9)1507-1510(2008).

11. N. Agata, and H. Karolina. "Brain - Computer interface based on Steady State Visual Evoked Potentials (SSVEP). International Carpathian Control Conference. IEEE, pp.251 - 254(2013).

12. M. P. Xu, L. X. Zhang, M. Dong, et al. A hybrid paradigm based on SSVEP blocking and P300 features of brain computer interface. Journal of Electronic Science, 41, pp. (11) 2247-2251(2013).

13. L. Zhao, Y. Sun, X. H. Goo, etal. Research on mobile phone dialing system steady state visual evoked potential of China biomedical engineering based on the, 32, pp. (2), 253-256(2013).

14. R. Gao, S. Y. Lu, Y. C. Zhang, etal. Brain computer interface technology research status and development trend of robot. Technology and application, 4, pp. 16-19(2008).

15. Y. F. Pan Clinical evoked potential, second edition. Beijing: People's Medical Publishing House, pp. 546-589(2000).(In Chinese) 